\title{
Asymptomatic Patients with Airflow Limitation are at Higher Risk of Postoperative Pulmonary Complications After Lung Surgeries: An Ambispective Cohort Study
}

\author{
Yang Wang' \\ Xiangyu Yin ${ }^{2}$ \\ Mengshuang Xie (D) ${ }^{3}$ \\ Qin Wang' \\ Wei Wang ${ }^{4}{ }^{4}$ \\ Wenbo $\mathrm{Yi}^{\mathrm{I}}$ \\ Jingui $\mathrm{Yu}^{\prime}$
}

'Department of Anesthesiology, Qilu Hospital, Cheeloo College of Medicine, Shandong University, Jinan, Shandong, 2500 I2, China; ${ }^{2}$ Department of Joint Surgery, The Second Hospital, Cheeloo College of Medicine, Shandong University, Jinan, Shandong, 250033,

China; ${ }^{3}$ Department of Geriatric

Medicine, Qilu Hospital, Cheeloo College of Medicine, Shandong University, Jinan, Shandong, 2500I2, China; ${ }^{4}$ Department of Pulmonary and Critical Care Medicine, School of Medicine, Cheeloo College of Medicine, Shandong University, Jinan, Shandong, 2500I2, China
Correspondence: Jingui Yu

Department of Anesthesiology, Qilu Hospital, Cheeloo College of Medicine, Shandong University, 107 Wenhua West Road, Jinan, Shandong, 2500I2, China

Tel +8618560086868

Fax +8653182166458

Email yujinguil 109@I26.com
Purpose: With the use of pulmonary function tests (PFTs) as a preoperative examination, it is not rare to screen out airflow limitation (AFL) in asymptomatic patients undergoing lung surgeries. This study aims to elucidate whether there is a difference in the prevalence and prognosis of postoperative pulmonary complications (PPCs) between asymptomatic patients with newly diagnosed AFL and the normal population undergoing lung surgeries.

Patients and Methods: The medical records of asymptomatic patients undergoing lung surgeries who were spirometrically diagnosed with AFL between January and October 2017 were collected in Qilu hospital. These patients were subsequently followed up until February 2021. The diagnosis of PPCs was based on a recommended consensus definition. The incidence of PPCs between the newly diagnosed AFL group and the normal group was compared and a propensity score-matched analysis (PSM) was performed. The survival analysis was performed to investigate the long-term prognosis of the two groups.

Results: Overall, 535 asymptomatic subjects were recruited and 126 subjects (11.4\%) were spirometrically diagnosed as AFL. The incidence of PPCs was significantly higher in the newly diagnosed AFL group than in the normal population (28.6\%VS $14.4 \%, \mathrm{P}<0.001)$, especially in the $\mathrm{FEV}_{1} / \mathrm{FVC} \leq 65 \%$ group $(\mathrm{P}<0.001)$, which were all confirmed by PSM analysis. Furthermore, these patients were at a higher risk of ICU admissions $(\mathrm{P}<0.001)$ and 90 -day hospital readmissions secondary to PPCs $(\mathrm{P}<0.001)$. No significant differences were found in the overall, in-hospital and 90-day mortality between the AFL group and the normal group ( $\mathrm{P}$ values $>0.05$ ).

Conclusion: Asymptomatic patients with AFL are at higher risk of PPCs than the general population after lung surgeries, along with an increase in ICU admissions and 90-day hospital readmissions secondary to PPCs. Although these patients tended to report worse current conditions, they were similar in the in-hospital, 90-day and overall mortality during the follow-up.

Keywords: pulmonary function tests, newly diagnosed airflow limitation, propensity scorematched analysis, postoperative outcomes

\section{Introduction}

As a recommended preoperative examination, pulmonary function tests (PFTs) have been widely performed to predict the incidence of perioperative complications as well as to determine the optimal treatment or surgical method, especially in patients undergoing lung surgeries. ${ }^{1,2}$ Along with the routine use of PFTs, it is not rare to screen out airflow limitation (AFL) in asymptomatic patients who have few respiratory symptoms and no diagnosed chronic cardiopulmonary diseases. 
Previous evidence has shown that the incidence of newly diagnosed AFL is far more higher than expected. ${ }^{3-7}$ Additionally, GOLD (Global Initiative for Chronic Obstructive Lung Disease) guidelines have indicated that never smokers with chronic airflow limitation may have no increased risk of lung cancer or cardiovascular

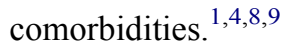

Postoperative pulmonary complications (PPCs), whose majority are postoperative pneumonia (POP) and respiratory failure, are common and frequently life-threatening in patients who have undergone lung surgeries, ${ }^{10-13}$ with an incidence ranging from $9 \%$ to $40 \%$ due to the lack of recognized definitions. ${ }^{14,15}$ Currently, PPCs have been acknowledged as the leading cause of postoperative morbidity and mortality, associated with additional costs of health care. ${ }^{13,15,16}$ Therefore, identifying potent patients at high risk for PPCs is critical to benefiting those patients and thus lowering health costs. As a verified risk factor for the development of PPCs, the presence of chronic obstructive pulmonary diseases (COPD) has a great impact on the prevalence and prognosis of PPCs. ${ }^{15,17,18}$ It's noted that patients with COPD may result in an increased risk of pneumonia and mortality from respiratory failure with the progression of the disease. ${ }^{1,49}$ Preoperative spirometry tests have indicated the presence of AFL in some asymptomatic patients undergoing lung surgeries. However, few attempts have been made to investigate whether these patients are at higher risk of PPCs than the general population after lung surgeries, especially in China. This study aims to elucidate whether there's a difference in the prevalence of PPCs between asymptomatic patients with newly-diagnosed AFL and the normal population undergoing lung surgeries in China. It should be noted that the group of patients without AFL was defined as "normal population". Furthermore, we followed up these patients and evaluated whether the development of AFL had an impact on the longterm life quality and survival after lung surgeries.

\section{Materials and Methods Study Subjects/Population}

This cohort study was conducted between November 2018 and February 2021 in a Chinese tertiary medical center (Qilu Hospital, Shandong University). The medical records of patients who underwent lung surgeries between January and October 2017 were reviewed from a detailed database. Patients who met the following inclusion criteria were included: 1) age between 18 and 80 years old; 2) preoperative PFTs were performed and interpreted strictly under American
Thoracic Society (ATS)/European Respiratory Society (ERS) guidelines; 3 ) the lung surgery was performed under general anesthesia with single lung ventilation. The medical data of 1102 subjects that met the inclusion criteria were systematically reviewed and collected. The exclusion criteria included: 1) self-reported doctor diagnosis of cardiopulmonary diseases, especially the medical history of asthma, chronic bronchitis or emphysema; 2) recent acute respiratory symptoms, particularly cough, phlegm producing or dyspnea in the last 14 days; 3) unsuitable surgical candidates, those who were either physically or mentally intolerant of surgeries; 4) information incomplete or data missing. The study was conducted in accordance with the Declaration of Helsinki. No intervention was performed, and all data in this study were fully anonymized. Written informed consent was obtained from each patient. The study protocol was approved by the Medical Ethics Committee of Qilu Hospital of Shandong University (No. 2018 (143)) and registered at the Chinese clinical trial registry (ChiCTR1800019394) in advance.

\section{Definitions}

Newly diagnosed AFL was spirometrically defined as a previously undiagnosed forced expiratory volume in the first second to forced vital capacity ratio $\left(\mathrm{FEV}_{1} / \mathrm{FVC}\right)$ $<70 \%$ in the above subjects following the GOLD guidelines. ${ }^{1,8}$ All the PFTs were routinely performed under strict quality control following the American Thoracic Society (ATS) guidelines in all the above thoracic surgical candidates. The remaining subjects were allocated to the normal group.

Based on a recommended consensus definition, ${ }^{14}$ only respiratory diagnoses that shared common pathophysiological mechanisms were identified as PPCs, which included: 1) atelectasis detected on computed tomography or chest radiograph; 2) pneumonia using US Centers for Disease Control criteria; 3) Acute Respiratory Distress Syndrome using Berlin consensus definition; 4) pulmonary aspiration (a clear clinical history and radiological evidences are essential for the diagnosis). ${ }^{12}$ As all the subjects were performed lung surgeries with single lung ventilation, suspected atelectasis without imaging confirmation was not recognized as PPCs.

\section{Data Collection and Follow-Up}

The perioperative data of the enrolled subjects were collected, which included: the demographic characteristics, smoking status, comorbidities, American Society of Anesthesiologists (ASA) class, spirometry results, surgical 
procedure, surgical method, pathology, analgesia and detailed postoperative outcomes. All the postoperative complications which occurred before discharge, the total length of stay (LOS) in hospital after the date of surgery, intensive care unit (ICU) admission and in-hospital mortality were also recorded in detail.

All patients were annually followed up for overall survival (OS). All the follow-up data were achieved using a telephone questionnaire. The 90-day readmission to hospital secondary to PPCs, current status, ongoing treatment and mortality were enrolled.

\section{Statistical Analysis}

For categorical data, data were expressed as counts (proportion) and compared using the chi-square or Fisher's exact tests. The Shapiro-Wilk test was used to test the assumption of normality. For continuous variables, data were expressed as mean \pm standard deviation (SD) or medians and compared using Student t or Mann-Whitney $U$-tests in accordance with their distributions, respectively. A stepwise multivariate binary logistic regression analysis was performed to identify the independent predictors of PPCs.

A propensity score-matched analysis (PSM) was performed using a multivariable logistic regression model based on the following cofounders: sex, age, BMI, smoking status, Brinkman Index $(\mathrm{BI}=$ cigarette packs smoked per day times years of smoking), diabetes mellitus (DM), hypertension, coronary heart disease (CHD), ischemic cerebrovascular disease (ICVD), ASA class, surgical procedure, surgical method, pathology and analgesia. Pairs of patients with normal spirometry or newly diagnosed AFL were derived using 1:1 greedy nearest neighbor matching within a propensity score of 0.02 . This statistical strategy resulted in 121 matched pairs. Comparisons between the matched groups were performed with paired $t$-test or Wilcoxon rank sum test for continuous variables and $\mathrm{McNemar}$ test for categorical variables.

The Kaplan-Meier method was used to perform cumulative analyses and differences between groups were assessed with the Log rank test. All the results were considered significant at $\mathrm{P}$ value $<0.05$. All the statistical analyses were performed using IBM SPSS Statistics (version 25.0; IBM, Armonk, NY, USA).

\section{Results}

Overall, the medical records of 1102 patients who underwent lung surgeries between January and October 2017 were reviewed. A total of 535 cases were enrolled and 126 subjects (11.4\%) were spirometrically diagnosed as AFL (Figure 1). According to the assessment tool recommended by GOLD, ${ }^{1,8}$ 74 cases $(58.7 \%)$ were classified into grade 1, group A (mild), 49 cases (38.9\%) into grade 2, group C (moderate) and 3 cases (2.4\%) into grade 3 , group C (severe). As is shown in Table 1, there were significant differences in sex, age and the extent of cigarette exposure (both smoking status and BI)

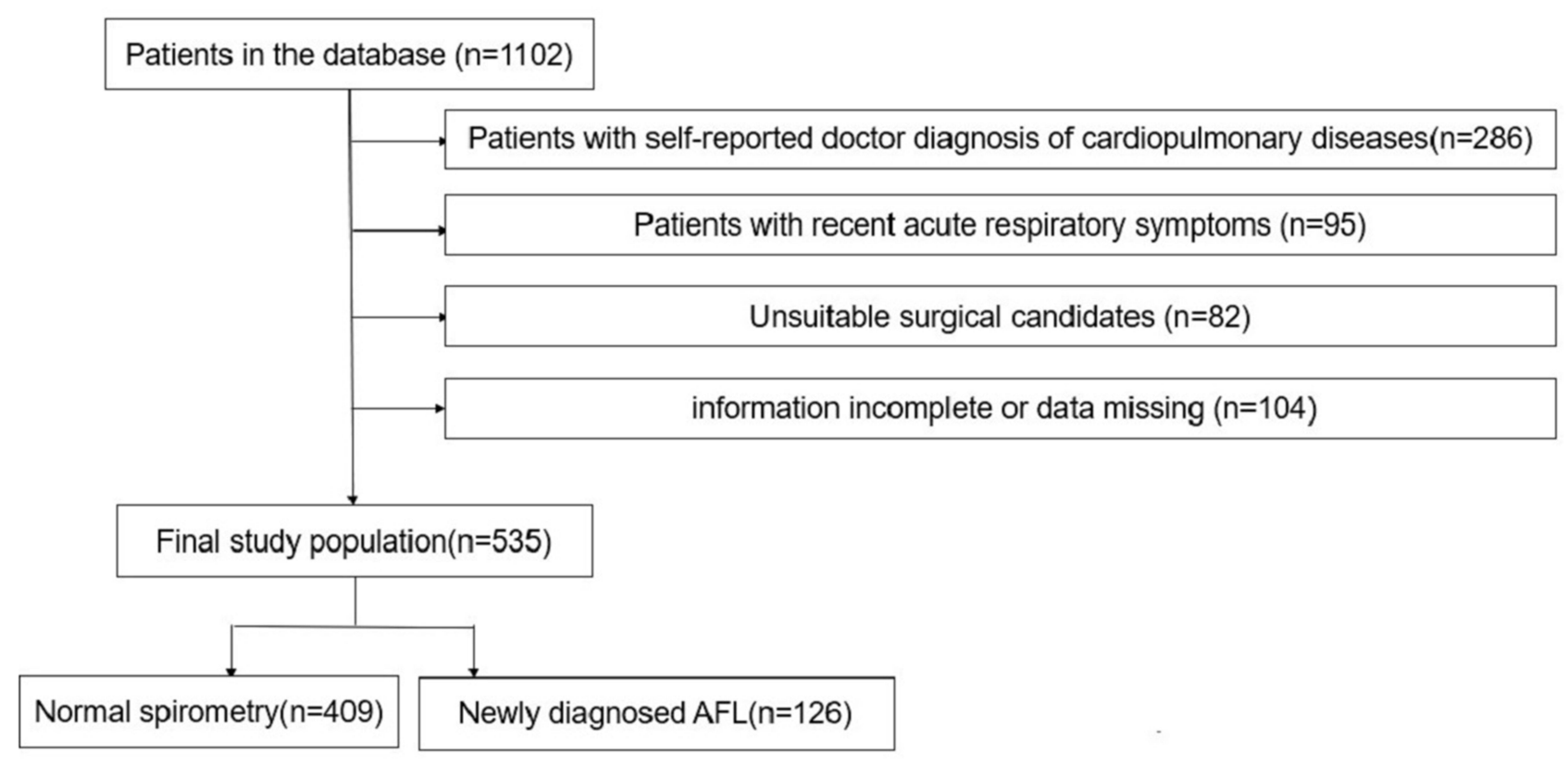

Figure I Flowchart of selection of the study population. Abbreviation: AFL, airflow limitation. 
Table I Baseline Characteristics of Study Population Prior to Propensity Score-Matching Analysis

\begin{tabular}{|c|c|c|c|c|c|c|c|}
\hline & \multirow{2}{*}{$\begin{array}{l}\text { Total } \\
(n=535)\end{array}$} & \multirow{2}{*}{$\begin{array}{l}\text { Normal } \\
\text { Spirometry } \\
(n=409)\end{array}$} & \multicolumn{4}{|c|}{ Newly Diagnosed AFL } & \multirow[t]{2}{*}{ P-value } \\
\hline & & & Total $(n=126)$ & $\begin{array}{l}\text { Mild } \\
\text { (Group } A, n=74 \text { ) }\end{array}$ & $\begin{array}{l}\text { Moderate to } \\
\text { Severe } \\
\text { (Group C, } n=52 \text { ) }\end{array}$ & P-value & \\
\hline Sex, male & $310(57.9)$ & $210(51.3)$ & $100(79.4)$ & $56(75.7)$ & $44(84.6)$ & 0.222 & $<0.001$ \\
\hline Age, years & $60(53-65)$ & $59(52-64)$ & $62(55.75-68)$ & $60.45 \pm 9.02$ & $62.33 \pm 9.93$ & 0.271 & $<0.001$ \\
\hline BMI, $\mathrm{kg} / \mathrm{m}^{2}$ & $25.08 \pm 3.39$ & $25.19 \pm 3.34$ & $24.74 \pm 3.56$ & $25.23 \pm 3.89$ & $24.05 \pm 2.91$ & 0.055 & 0.193 \\
\hline \multicolumn{8}{|l|}{ Smoking status } \\
\hline Never & $329(61.5)$ & $282(68.9)$ & $47(37.3)$ & $32(43.2)$ & $15(28.8)$ & 0.202 & $<0.001$ \\
\hline Ex-smoker & $16(3.0)$ & $10(2.4)$ & $6(4.8)$ & $4(5.4)$ & $2(3.8)$ & & \\
\hline Current-smoker & 190(35.5) & $117(28.6)$ & $73(57.9)$ & $38(51.4)$ & $35(67.3)$ & & \\
\hline $\mathrm{BI}$ & $0(0-600)$ & $0(0-375)$ & $400(0-800)$ & $200(0-725)$ & $200(0-700)$ & 0.021 & $<0.001$ \\
\hline$\geq 400$ & $150(28.0)$ & $88(21.5)$ & $64(50.8)$ & $29(39.2)$ & $33(63.5)$ & 0.007 & $<0.001$ \\
\hline$<400$ & $385(72.0)$ & $321(78.5)$ & $62(49.2)$ & $45(60.8)$ & $19(36.5)$ & & \\
\hline \multicolumn{8}{|l|}{ Comorbidity } \\
\hline DM & $71(13.3)$ & $52(12.7)$ & $19(15.1)$ & $10(13.5)$ & $9(17.3)$ & 0.558 & 0.494 \\
\hline Hypertension & $124(23.2)$ & $86(21.0)$ & $38(30.2)$ & $19(25.7)$ & $19(36.5)$ & 0.191 & 0.034 \\
\hline $\mathrm{CHD}$ & $55(10.3)$ & $41(10.0)$ & $14(11.1)$ & $8(10.8)$ & $6(11.5)$ & 0.898 & 0.725 \\
\hline ICVD & $22(4.1)$ & $18(4.4)$ & $4(3.2)$ & $3(4.1)$ & $\mathrm{I}(1.9)$ & 0.502 & 0.544 \\
\hline \multicolumn{8}{|l|}{ ASA class } \\
\hline I, II & $522(97.4)$ & $400(97.8)$ & $122(96.8)$ & 73(98.6) & $49(94.2)$ & 0.305 & 0.516 \\
\hline III & $13(2.4)$ & $9(2.2)$ & $4(3.2)$ & $\mathrm{I}(\mathrm{l} .4)$ & $3(5.8)$ & & \\
\hline \multicolumn{8}{|l|}{ Perioperative pulmonary } \\
\hline function & & & & & & & \\
\hline $\mathrm{FEV}_{1} / \mathrm{FVC}$ & $75.77 \pm 8.09$ & $79.19 \pm 5.11$ & $64.67 \pm 5.66$ & $67.11 \pm 2.62$ & $61.20 \pm 6.92$ & $<0.001$ & $<0.001$ \\
\hline FEV,$\%$ pred & $100.08 \pm 19.05$ & $104.99 \pm 16.84$ & $84.13 \pm 16.99$ & $94.60 \pm 12.80$ & $69.22 \pm 9.36$ & $<0.001$ & $<0.001$ \\
\hline \multicolumn{8}{|l|}{ Surgical procedure } \\
\hline Lobectomy & $454(84.9)$ & $346(84.6)$ & 108(85.7) & $68(91.9)$ & $40(76.9)$ & 0.023 & 0.406 \\
\hline Subsegmentectomy/ wedge & $55(10.3)$ & $44(10.8)$ & $11(8.7)$ & $2(2.7)$ & $9(17.3)$ & & \\
\hline Pneumonectomy & $17(3.2)$ & $14(3.4)$ & $3(2.4)$ & $\mathrm{I}(1.4)$ & $2(3.8)$ & & \\
\hline Others & $9(1.7)$ & $5(1.2)$ & $4(3.2)$ & $3(4.1)$ & $1(1.9)$ & & \\
\hline \multicolumn{8}{|l|}{ Surgical method } \\
\hline VATS & $445(83.2)$ & $349(85.3)$ & $96(76.2)$ & $56(75.7)$ & $40(76.9)$ & 0.871 & 0.016 \\
\hline Open surgery & $90(16.8)$ & $60(14.7)$ & $30(23.8)$ & $18(24.3)$ & $12(23.1)$ & & \\
\hline \multicolumn{8}{|l|}{ Pathology } \\
\hline \multicolumn{8}{|l|}{ NSCLC } \\
\hline Adenocarcinoma & $356(66.5)$ & $273(66.7)$ & $83(65.9)$ & $49(66.2)$ & $34(64.5)$ & 0.529 & 0.317 \\
\hline Squamous & $106(19.8)$ & $75(18.3)$ & $31(24.6)$ & $20(27.0)$ & $11(21.2)$ & & \\
\hline Other NSCLC & $9(1.7)$ & $6(1.5)$ & $3(2.4)$ & $2(2.7)$ & $\mathrm{I}(1.9)$ & & \\
\hline Metastatic disease & $10(1.9)$ & $8(2.0)$ & $2(1.6)$ & $\mathrm{I}(1.4)$ & $\mathrm{I}(1.9)$ & & \\
\hline Benign tumor & $4 \mathrm{I}(7.7)$ & $36(8.8)$ & $5(4.0)$ & $2(2.7)$ & $3(5.8)$ & & \\
\hline Others & $13(2.4)$ & $\mathrm{II}(2.7)$ & $2(1.6)$ & $0(0.0)$ & $2(3.8)$ & & \\
\hline \multicolumn{8}{|l|}{ Analgesia } \\
\hline PCA only & $346(64.7)$ & $265(64.8)$ & $81(64.3)$ & $44(59.5)$ & $37(71.2)$ & 0.382 & 0.930 \\
\hline Epidural +PCA & 165(30.8) & $125(30.6)$ & $40(31.7)$ & $27(36.5)$ & $13(25.0)$ & & \\
\hline Nerve block +PCA & $24(4.5)$ & $19(4.6)$ & $5(4.0)$ & $3(4.1)$ & $2(3.8)$ & & \\
\hline
\end{tabular}

Notes: Data are expressed as mean \pm SD or medians with an interquartile range (25-75).

Abbreviations: BMI, body mass index; BI, Brinkman Index; ICVD, ischemic cerebrovascular disease; DM, diabetes mellitus; CHD, coronary heart disease; ASA, American Society of Anesthesiologists; VATS, video-assisted thoracoscopic surgery; NSCLC, non-small cell lung cancer; PCA, patient-controlled analgesia. 
between subjects with AFL and normal spirometry. However, baseline characteristics before and after PSM analysis, respecno significant differences were found between group $\mathrm{A}$ and tively. After PSM analysis, both groups were well matched in group $\mathrm{C}$, except for $\mathrm{BI}(\mathrm{P}<0.05)$. Tables 1 and 2 show the all the baseline parameters concerned ( $\mathrm{P}$ values $>0.05$ ).

Table 2 Baseline Characteristics of Study Patients After Propensity Score-Match Analysis

\begin{tabular}{|c|c|c|c|}
\hline Total $(n=242)$ & Normal Spirometry $(n=|2|)$ & Newly Diagnosed AFL $(n=|2|)$ & P-value \\
\hline Sex, male & $97(80.2)$ & $95(78.5)$ & 0.815 \\
\hline Age, years & $62.07 \pm 8.10$ & $60.93 \pm 9.42$ & 0.276 \\
\hline BMI, $\mathrm{kg} / \mathrm{m}^{2}$ & $24.78 \pm 3.03$ & $24.74 \pm 3.48$ & 0.923 \\
\hline \multicolumn{4}{|l|}{ Smoking status } \\
\hline Never & $55(45.5)$ & $46(38.0)$ & 0.425 \\
\hline Ex-smoker & $6(5.0)$ & $6(5.0)$ & \\
\hline Current-smoker & $60(49.6)$ & $69(57.0)$ & \\
\hline Brinkman Index & $200(0-800)$ & $400(0-800)$ & 0.915 \\
\hline$\geq 400$ & $50(41.3)$ & $58(47.9)$ & 0.229 \\
\hline$<400$ & $71(58.7)$ & $63(52.1)$ & \\
\hline \multicolumn{4}{|l|}{ Comorbidity } \\
\hline DM & $23(19.0)$ & $19(15.7)$ & 0.627 \\
\hline Hypertension & $30(24.8)$ & $36(29.8)$ & 0.451 \\
\hline CHD & $21(17.4)$ & $14(11.6)$ & 0.281 \\
\hline ICVD & $4(3.3)$ & $4(3.3)$ & 1.000 \\
\hline \multicolumn{4}{|l|}{ ASA class } \\
\hline I, II & $119(98.3)$ & $117(96.7)$ & 0.687 \\
\hline III & $2(1.7)$ & $4(3.3)$ & \\
\hline \multicolumn{4}{|c|}{ Perioperative pulmonary function } \\
\hline $\mathrm{FEV}_{\mathrm{I}} / \mathrm{FVC}$ & $74.24 \pm 3.08$ & $64.57 \pm 5.73$ & $<0.001$ \\
\hline FEV ${ }_{1} \%$ pred & $99.62 \pm 26.50$ & $83.73 \pm 17.17$ & $<0.001$ \\
\hline \multicolumn{4}{|l|}{ Surgical procedure } \\
\hline Lobectomy & $104(86.0)$ & $103(85.1)$ & 0.549 \\
\hline Subsegmentectomy/wedge & $10(8.3)$ & $1 \mathrm{l}(9.1)$ & \\
\hline Pneumonectomy & $5(4.1)$ & $3(2.5)$ & \\
\hline Others & $2(1.7)$ & $4(3.3)$ & \\
\hline \multicolumn{4}{|l|}{ Surgical method } \\
\hline VATS & $96(79.3)$ & $93(76.9)$ & 0.749 \\
\hline Open surgery & $25(20.7)$ & $28(23.1)$ & \\
\hline \multicolumn{4}{|l|}{ Pathology } \\
\hline \multicolumn{4}{|l|}{ NSCLC } \\
\hline Adenocarcinoma & $76(62.8)$ & $80(66.1)$ & 0.780 \\
\hline Squamous & $31(25.6)$ & $29(24.0)$ & \\
\hline Other NSCLC & $3(2.5)$ & $3(2.5)$ & \\
\hline Metastatic disease & $\mathrm{I}(0.8)$ & $2(1.7)$ & \\
\hline Benign tumor & $9(7.4)$ & $5(4.1)$ & \\
\hline Others & $\mathrm{I}(0.8)$ & $2(1.7)$ & \\
\hline \multicolumn{4}{|l|}{ Analgesia } \\
\hline PCA only & $79(65.3)$ & $78(64.5)$ & 0.412 \\
\hline Epidural +PCA & $4 I(33.9)$ & $38(31.4)$ & \\
\hline Nerve block +PCA & $\mathrm{I}(0.8)$ & $5(4.1)$ & \\
\hline
\end{tabular}

Notes: Data are expressed as mean \pm SD or medians with an interquartile range (25-75).

Abbreviations: BMI, body mass index; BI, Brinkman Index; ICVD, ischemic cerebrovascular disease; DM, diabetes mellitus; CHD, coronary heart disease; ASA, American Society of Anesthesiologists; VATS, video-assisted thoracoscopic surgery; NSCLC, non-small cell lung cancer; PCA, patient-controlled analgesia. 
The stepwise multivariate logistic regression indicated that age and AFL were independent predictors of PPCs (Table 3). Compared with the normal population, patients with newly diagnosed AFL were 1.79 times more likely to develop PPCs $(95 \%$ CI 1.07 to $3.00, \mathrm{P}=0.026)$. Meanwhile, elderly patients were 1.04 times more likely to develop PPCs in comparison with the normal group (95\% CI 1.01 to $1.07, \mathrm{P}=0.003$ ).

Based on the above-mentioned consensus definition, a total of 95 subjects (17.7\%) had the clinical evidence of PPCs, with a significant increase in the newly diagnosed AFL group (28.6\%VS $14.4 \%, \mathrm{P}<0.001)$. As is shown in Table 4, the majority of PPCs were POP $(n=82,15.3 \%)$, followed by respiratory failure $(\mathrm{n}=7,1.3 \%)$, atelectasis $(\mathrm{n}=5,0.9 \%)$ and aspiration $(n=1,0.2 \%)$. Patients in the newly diagnosed AFL group had a longer LOS after surgery (10 (9 to 11) vs 11 (9 to 13.25), $\mathrm{P}<0.001$ ), significantly higher rates of ICU admissions $(15.1 \%$ vs $4.9 \%, \mathrm{P}<0.001)$, tracheal intubation ( $1.6 \%$ vs $1.0 \%, \mathrm{P}=0.001$ ) and 90 -day hospital readmissions secondary to PPCs $(7.9 \%$ vs $0.7 \%, \mathrm{P}<0.001)$. Furthermore, among the AFL group, the prevalence of PPCs remained significantly higher among those in the $\mathrm{FEV}_{1} / \mathrm{FVC} \leq 65 \%$ group with the decline in $\mathrm{FEV}_{1} / \mathrm{FVC}(\mathrm{P}<0.001)$, which was in accordance with the higher rates of ICU admissions $(\mathrm{P}=0.002)$, tracheal intubation $(\mathrm{P}=0.002)$ and 90 -day hospital readmissions secondary to PPCs $(\mathrm{P}=0.005)$.

Patients with AFL tended to suffer from a worse selfreported current status than the normal population $(\mathrm{P}=0.028$, Table 4). A total of 74 patients (13.8\%) died during follow-up, 18 cases were from the newly diagnosed AFL group, while the remaining 56 cases were from the normal group $(14.3 \%$ vs
$13.7 \%, \mathrm{P}=0.866$ ). Causes of death included PPC-related deaths $(4,0.7 \%)$, surgery-related deaths $(2,0.4 \%)$, cancerrelated deaths $(59,11.0 \%)$ and others $(9,1.7 \%)$. There were no significant differences in the overall mortality, in-hospital mortality, 90-day mortality and causes of death between the AFL group and the normal group (P values $>0.05$, Table 4). Furthermore, no significant differences were found with the decline of $\mathrm{FEV}_{1} / \mathrm{FVC}$ ratios concerning the above mortalities ( $P$ values $>0.05$, Table 4 ). All the patients were followed up for 41 to 49 months, the overall survival (cumulative survival rates) was not significantly different between the AFL group and the normal group before and after PSM analysis ( $\mathrm{P}=$ 0.781, Figure 2 and $P=0.092$, Figure 3).

After PSM analysis, the prevalence of PPCs in the AFL group was still significantly higher than that in the normal group ( $\mathrm{P}<0.001)$, along with an increase in ICU admissions $(\mathrm{P}=0.049)$ and 90 -day hospital readmissions secondary to PPCs $(\mathrm{P}=0.021$, Table 5). However, there was no significant difference in the mean LOS between the two groups after PSM analysis $(\mathrm{P}=0.067$, Table 5$)$.

\section{Discussion}

A total of up to $11.4 \%$ (126/535) patients undergoing lung surgeries were spirometrically diagnosed as AFL using the fixed ratio $\left(\mathrm{FEV}_{1} / \mathrm{FVC}<70 \%\right)$ though preoperative spirometry and the incidence were in accordance with previous studies. ${ }^{3,4,6,19}$ Generally, elderly males with higher levels of tobacco exposure tended to suffer from AFL. Our study confirmed that asymptomatic patients with AFL were at higher risk of PPCs than the general population in those who had undergone lung surgeries, accompanied by an

Table 3 Multivariate Logistic Regression Analysis to Assess Predictors of PPCs

\begin{tabular}{|c|c|c|c|c|c|}
\hline Variable & $\boldsymbol{\beta}$ & Wald & P-value & OR & $95 \% \mathrm{Cl}$ \\
\hline Age & 0.040 & 8.627 & 0.003 & 1.041 & $1.013-1.069$ \\
\hline Male & 0.057 & 0.040 & $0.84 I$ & 1.059 & $0.604-1.856$ \\
\hline ASA III & -0.843 & 1.705 & 0.192 & 0.430 & $0.121-1.526$ \\
\hline Newly diagnosed AFL & 0.585 & 4.955 & 0.026 & 1.794 & $1.072-3.002$ \\
\hline $\mathrm{BI} \geq 400$ & 0.414 & 2.191 & 0.139 & 1.513 & $0.874-2.617$ \\
\hline Lobectomy & & & 0.803 & & \\
\hline Subsegmentectomy/wedge & 0.220 & $0.36 \mathrm{I}$ & 0.548 & 1.246 & $0.607-2.557$ \\
\hline Pneumectomy & 0.492 & 0.710 & 0.400 & 1.635 & $0.52 I-5.131$ \\
\hline Other surgical procedures & 0.056 & 0.004 & 0.947 & 1.508 & $0.203-5.500$ \\
\hline
\end{tabular}

Abbreviations: ASA, American Society of Anesthesiologists; AFL, airflow limitation; BI, Brinkman Index; OR, odds ratio; Cl, confidence index. 
Table 4 Postoperative Outcomes Prior to Propensity Score-Match Analysis

\begin{tabular}{|c|c|c|c|c|c|c|c|}
\hline & \multirow{2}{*}{$\begin{array}{l}\text { Total } \\
(n=535)\end{array}$} & \multirow{2}{*}{$\begin{array}{l}\text { Normal } \\
\text { Spirometry } \\
(\mathrm{n}=409)\end{array}$} & \multicolumn{4}{|c|}{ Newly Diagnosed AFL } & \multirow[t]{2}{*}{ P-value } \\
\hline & & & $\begin{array}{l}\text { Total } \\
(n=\mid 26)\end{array}$ & $\begin{array}{l}65 \%<\mathrm{FEV}_{1} / \mathrm{FVC}< \\
70 \%(\mathrm{n}=80)\end{array}$ & $\begin{array}{l}\text { FEV }_{1} / \mathrm{FVC} \\
\leq 65 \%(n=46)\end{array}$ & P-value & \\
\hline Mean LOS (days) & $10(9-12)$ & $10(9-11)$ & $11(9-13.25)$ & $10(9-13)$ & $11(8-15)$ & 0.177 & $<0.001$ \\
\hline \multicolumn{8}{|l|}{ Overall in-hospital complications } \\
\hline PPCs (at least one) & $95(17.8)$ & $59(14.4)$ & $36(28.6)$ & $15(18.8)$ & $21(45.7)$ & $<0.001$ & $<0.001$ \\
\hline POP & $82(15.3)$ & $51(12.5)$ & $31(24.6)$ & $14(17.5)$ & $17(37.0)$ & $<0.001$ & $<0.001$ \\
\hline Respiratory failure & $7(1.3)$ & $3(0.7)$ & $4(3.2)$ & $0(0)$ & $4(8.7)$ & & \\
\hline Atelectasis & $5(0.9)$ & $4(1.0)$ & $\mathrm{I}(0.8)$ & $\mathrm{I}(1.3)$ & $0(0)$ & & \\
\hline Pulmonary aspiration & $I(0.2)$ & $I(0.2)$ & $0(0)$ & $0(0)$ & $0(0)$ & & \\
\hline Atrial fibrillation & $3(0.6)$ & $2(0.5)$ & $I(0.8)$ & $\mathrm{I}(1.3)$ & $0(0)$ & 0.446 & 0.689 \\
\hline Acute myocardial infarction & $2(0.4)$ & $I(0.2)$ & $\mathrm{I}(0.8)$ & $0(0)$ & $\mathrm{I}(2.2)$ & 0.186 & 0.377 \\
\hline Aortic dissection & $I(0.2)$ & $I(0.2)$ & $0(0)$ & $0(0)$ & $0(0)$ & 1.000 & 0.578 \\
\hline Bronchopleural fistula & $I(0.2)$ & $0(0)$ & $\mathrm{I}(0.8)$ & $0(0)$ & $I(2.2)$ & 0.186 & 0.072 \\
\hline Pulmonary embolism & $I(0.2)$ & $\mathrm{I}(0.2)$ & $0(0)$ & $0(0)$ & $0(0)$ & 1.000 & 0.578 \\
\hline ICU admissions & $39(7.3)$ & $20(4.9)$ & $19(15.1)$ & $6(7.5)$ & $13(28.3)$ & 0.002 & $<0.001$ \\
\hline Tracheal intubation & $6(1.1)$ & $4(1.0)$ & $2(1.6)$ & $0(0)$ & $2(4.3)$ & 0.002 & 0.001 \\
\hline 90-day hospital readmissions secondary to PPCs & $14(2.6)$ & $4(0.7)$ & 10(7.9) & $2(2.5)$ & $8(17.4)$ & 0.005 & $<0.001$ \\
\hline \multicolumn{8}{|l|}{ Current status (quality of life) } \\
\hline In good condition & $370(69.2)$ & $283(69.2)$ & $87(69.0)$ & $58(72.5)$ & $29(63.0)$ & 0.435 & 0.028 \\
\hline Out of condition & $31(5.8)$ & $18(4.4)$ & $13(10.3)$ & $7(8.8)$ & $6(13.0)$ & & \\
\hline \multicolumn{8}{|l|}{ Mortality } \\
\hline Total & $74(13.8)$ & $56(13.7)$ & $18(14.3)$ & $9(11.3)$ & $9(19.6)$ & 0.164 & 0.866 \\
\hline In-hospital mortality & $4(0.7)$ & $3(0.7)$ & $I(0.8)$ & $0(0)$ & $\mathrm{I}(2.2)$ & & 0.944 \\
\hline 90-day mortality & $9(1.7)$ & $7(1.7)$ & $2(1.6)$ & $0(0)$ & $2(4.3)$ & & 0.924 \\
\hline \multicolumn{8}{|l|}{ Causes of death } \\
\hline PPCs & $4(0.7)$ & $3(0.7)$ & $I(0.8)$ & $0(0)$ & $1(2.2)$ & 0.186 & 0.944 \\
\hline Surgery-related & $2(0.4)$ & $\mathrm{I}(0.2)$ & $\mathrm{I}(0.8)$ & $0(0)$ & $\mathrm{I}(2.2)$ & & 0.377 \\
\hline Cancer-related & $59(11.0)$ & $46(11.2)$ & $13(10.3)$ & $7(8.8)$ & $6(13.0)$ & & 0.771 \\
\hline \multicolumn{8}{|l|}{ Others } \\
\hline Respiratory & $3(0.6)$ & $2(0.5)$ & $I(0.8)$ & $0(0)$ & $I(2.2)$ & & 0.689 \\
\hline Cardiovascular & $2(0.4)$ & $I(0.2)$ & $I(0.8)$ & $\mathrm{I}(1.3)$ & $0(0)$ & & 0.377 \\
\hline Others & $4(0.7)$ & $3(0.7)$ & $\mathrm{I}(0.8)$ & $\mathrm{I}(1.3)$ & $0(0)$ & & 0.944 \\
\hline
\end{tabular}

Notes: Data are expressed as medians with an interquartile range (25-75).

Abbreviations: PPC, postoperative pulmonary complications; AFL, airflow limitation; LOS, the total length of stay in hospital after the date of surgery; POP, postoperative pneumonia.

increase in ICU admissions and 90-day hospital readmissions secondary to PPCs both before and after PSM analysis.

GOLD guidelines recommended the use of postbronchodilator spirometry test and the fixed ratio as cutoff point (instead of lower limit of normal (LLN)) to confirm the presence of persistent airflow limitation. ${ }^{1,2,8}$ However, only pre-bronchodilator values were achieved in our study, similar to most published studies. Previous studies had shown that reversible obstruction accounted for approximately $35 \%$ and the corresponding post-bronchodilator $\mathrm{FEV}_{1} / \mathrm{FVC}$ ratios would return to the normal range (beyond $70 \%)^{20,21}$ On the other hand, it was reported that up to $93 \%$ individuals could be diagnosed as irreversible AFL if the cutoff value of prebronchodilator $\mathrm{FEV}_{1}$ $/ \mathrm{FVC}$ ratios were set as $65 \% .^{20,22}$ To avoid potential overestimation of AFL, we divided the newly diagnosed AFL group into two groups based on the decline of $\mathrm{FEV}_{1} / \mathrm{FVC}$ ratios (1. those lower than $65 \%$; 2 . those between $65 \%$ and $70 \%$ ) and made a comparison.

One highlight of the present study was the definition of PPCs we applied. Numerous postoperative pulmonary outcome measures were used in previous studies, but many were imprecise and poorly defined. The lack of recognized 


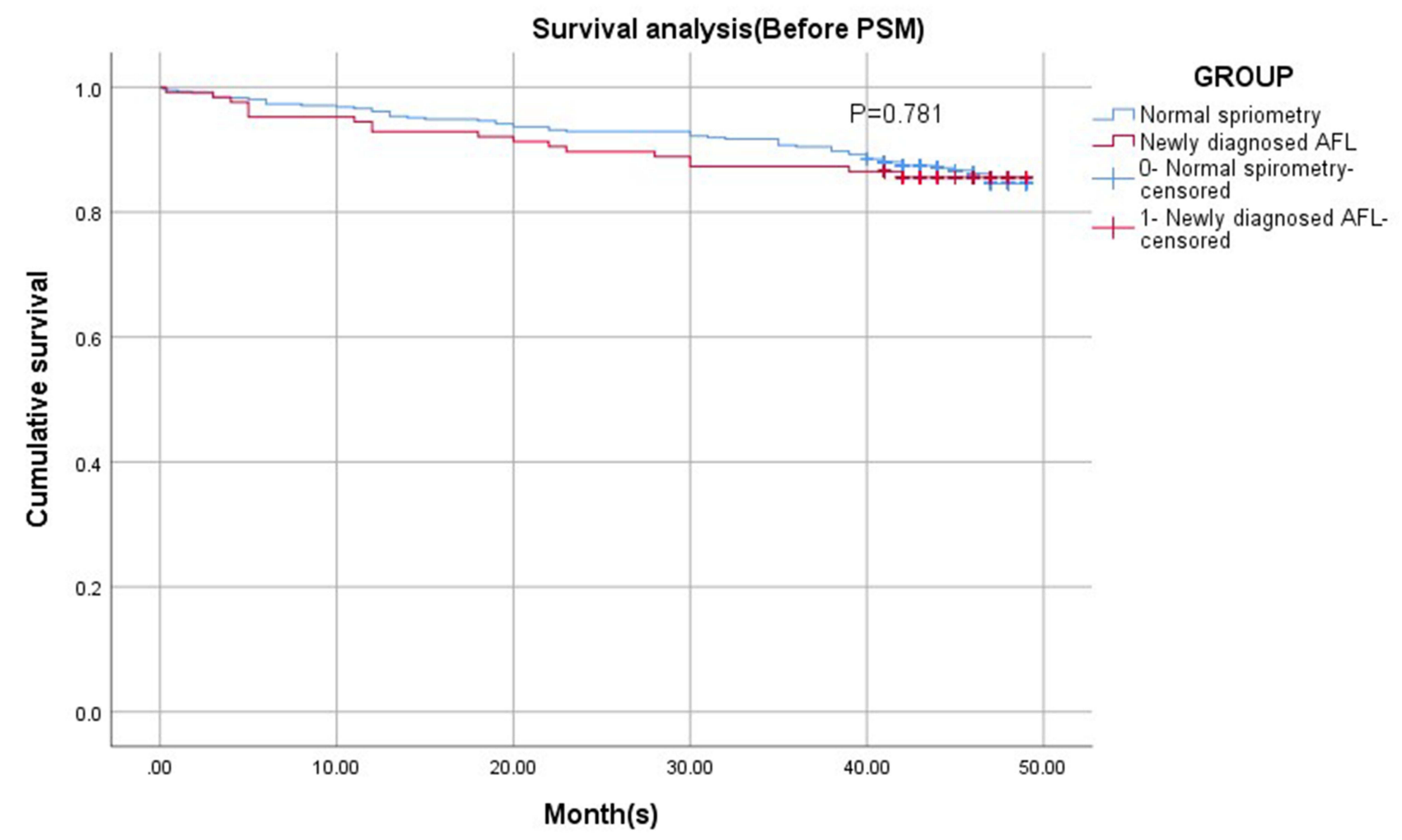

Figure 2 Kaplan-Meier cumulative event curves of all-cause death before propensity score-matching. Abbreviations: PSM, propensity score-matched analysis; AFL, airflow limitation.

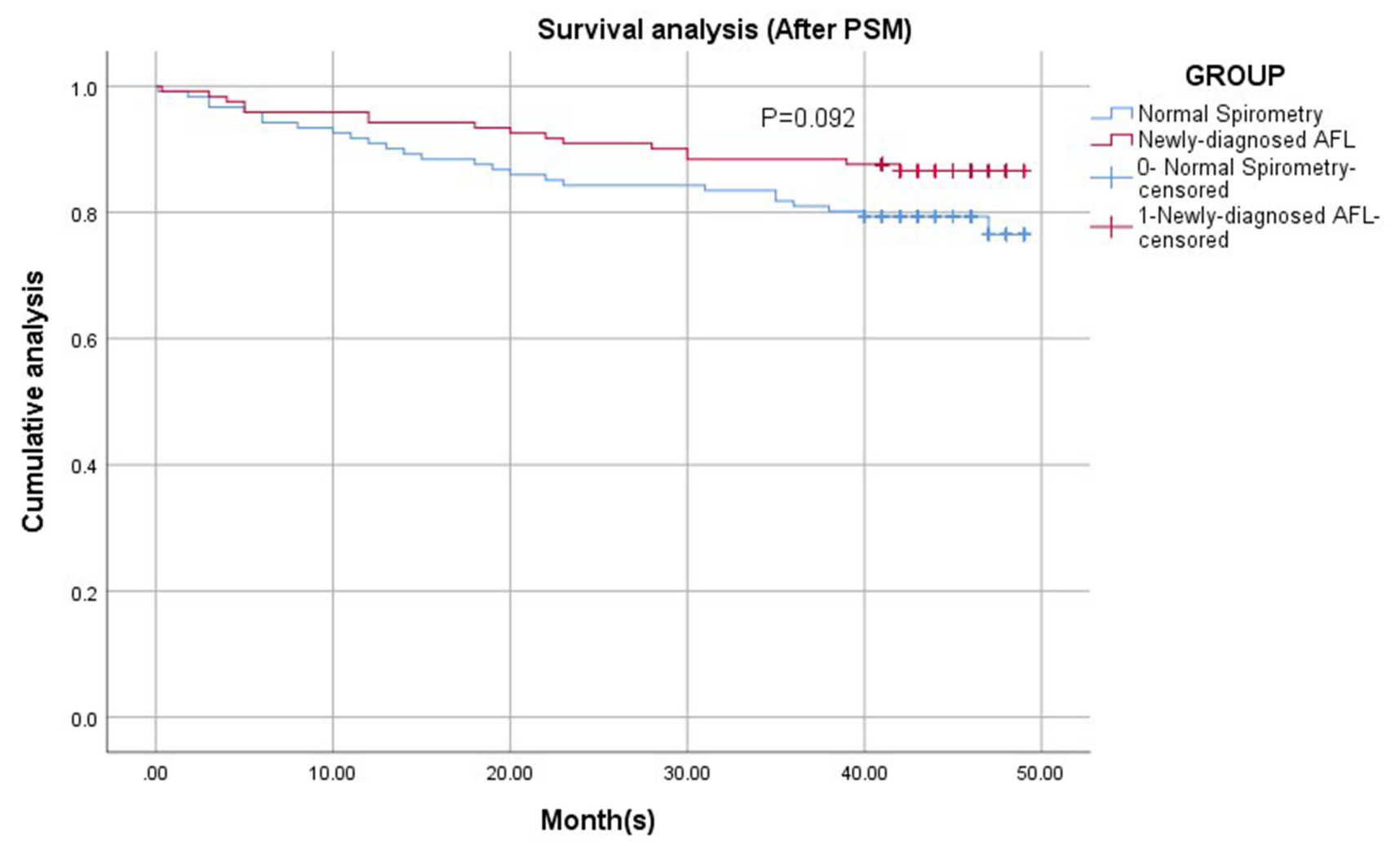

Figure 3 Kaplan-Meier cumulative event curves of all-cause death after propensity score-matching. Abbreviations: PSM, propensity score-matched analysis; AFL, airflow limitation. 
Table 5 Postoperative Outcomes After Propensity Score-Match Analysis

\begin{tabular}{|l|l|l|l|}
\hline & Normal Spirometry (n= I 2 I) & Newly Diagnosed AFL (n=I2 I) & P-value \\
\hline Mean LOS (days) & $10(9-12)$ & II (9-13) & 0.067 \\
\hline Overall in-hospital complications & & & \\
PPCs & $21(17.3)$ & $35(28.9)$ & $<0.001$ \\
ICU admissions & $9(7.4)$ & $18(14.8)$ & 0.049 \\
90-day hospital readmissions secondary to PPCs & $2(1.7)$ & $10(8.3)$ & 0.021 \\
\hline
\end{tabular}

Notes: Data are expressed as medians with an interquartile range (25-75).

Abbreviations: AFL, airflow limitation; LOS, the total length of stay in hospital after the date of surgery; PPC, postoperative pulmonary complications.

consensus definitions resulted in great differences in the prevalence of PPCs, which varied from $9 \%$ to $40 \%{ }^{14,15}$ After performing a systematic literature review and a three-stage Delphi consensus process, Abbott and his colleagues selected a set of recognized and precise definitions for perioperative complications. ${ }^{14}$ Our study took the lead to apply this set of recommended definitions to Chinese patients in order to improve our design and avoid misdiagnosis of PPCs.

Our study confirmed that the prevalence of PPCs, ICU admissions, tracheal intubation and 90-day hospital readmissions secondary to PPCs remained significantly higher in patients with newly diagnosed airflow limitation than the normal group, with the decline of $\mathrm{FEV}_{1} / \mathrm{FVC}$ ratio. Patients in the newly diagnosed group tended to have a longer LOS, but no significant difference was found after PSM analysis. Some patients in the matched pairs received postoperative chemotherapy and resulted in a prolonged LOS in the normal group, which could partly explain the above-mentioned discrepancy.

It is noteworthy that two patients with moderate-tosevere airflow limitation discharged without occurrence of PPCs, ICU admissions, prolonged LOS or 90-day readmissions after standardized preoperative inhalation treatment. Although both patients reported few preoperative respiratory symptoms, they were spirometrically diagnosed with moderate-to-severe airflow limitation. Although their lung function showed no obvious improvement after inhalation treatment, both short-term and longterm prognosis were desirable. This implied that standardized preoperative inhalation treatment might be taken into consideration as an effective way to reduce the incidence of PPCs and benefit potential patients. In future, more relevant RCTs (randomized controlled trial) are in urgent need to verify our assumptions.

Our study confirmed that newly diagnosed AFL was an independent predictor of PPCs in asymptomatic patients undergoing lung surgeries. Consistent with previous studies, age was also found to be associated with the development of PPCs, which indicated even elderly healthy patients were at higher risk of PPCs. ${ }^{12,15,23}$ However, when age was regarded as the predictor of PPCs, OR was only $1.04(95 \%$ CI 1.01 to $1.07, \mathrm{P}=0.003)$. One possible explanation is the absence of age stratification. In the meantime, larger sample size would be another solution. It is generally accepted that better functional preservation is accompanied by less reduction in the volume of surgery. Patients scheduled for pneumonectomy are considered as the most likely to suffer from PPCs, 30-day mortality and worse long-term prognosis. ${ }^{24-26}$ It's also demonstrated that segmentectomy is recommended for patients with a normal predicted postoperative $\mathrm{FEV}_{1}(\%$ ppoFEV $V_{1}$ ) while offers no functional advantages over lobectomy in patients with the $\%$ ppoFEV 1 under $70 \%{ }^{27}$ However, our study indicated that surgical procedures were not significantly associated with the incidence of PPCs, tracheal intubation or repeated hospitalization. The majority of patients $(454 / 535,84.9 \%)$ were indicated for lobectomy, while only very few patients underwent pneumonectomy $(17 / 535,3.2 \%)$. This bias might be a possible reason.

To our knowledge, few attempts have been made to investigate the long-term impact of airflow limitation after lung surgeries in China. Recent studies have shown that PPCs are predictors of both short-term and long-term postoperative outcomes and are associated with increased risk of ICU admissions and prolonged LOS. ${ }^{16,23}$ Sebastian and his colleagues indicated that the development of PPCs after thoracic surgery was associated with a worse longterm survival and these patients were more likely to die of non-cancer-related deaths. ${ }^{12}$ However, previous studies did not elucidate whether the development of AFL had an impact on the long-term quality of life and our study explored this issue. Although patients with AFL tended 
to report worse current condition than the normal population during the follow-up, they were similar in in-hospital, 90-day, overall mortality and cumulative survival rates. A large proportion of patients who underwent lung surgeries in our study were generally in good condition and admitted to hospital due to early detection of lung tumor after routine physical examinations. Most of them received clinical recovery after surgical removal and benefited from early diagnosis. This might account for our findings on the similarity in long-term survival between the AFL group and the normal population. In our view, PPC-related deaths (included in the non-cancer-related deaths) were more likely to occur during hospital stay and 90 days after surgery. However, it remains to be elucidated whether the sample size and multicenter trials would make a difference.

It is well documented that the incidence of newly diagnosed AFL is far more higher than expected. Relatively little is understood about these asymptomatic patients, and they definitely deserve more attention. Our study confirmed that the detection of AFL in asymptomatic patients undergoing lung surgeries was an effective way to identify potential patients at high risk for PPCs. According to our findings, we hypothesize that these asymptomatic surgical candidates would benefit from early diagnosis of AFL and early treatment (standardized preoperative inhalation treatment). If so, in order to reduce the incidence of PPCs, spirometry could be performed as a screening tool for patients at high risk and preoperative inhalation treatment could be recommended for asymptomatic patients with newly diagnosed AFL. It implied that this might be an effective way to benefit potential patients and lower medical costs in the long run. There is an urgent need for more well-designed clinical trials to test our hypothesis.

Our study had several limitations. As an ambispective study, some data were retrospectively achieved and the best choice would be RCT. PSM analysis was performed to minimize confounding bias in patient selection, but unknown confounders might exist. At present, PSM analysis could be an acceptable alternative and relative prospective RCTs are in high demand in future. Secondly, as a single-center study, we lacked the external validity required to support widespread changes in practice. Largescale, multicenter trials are in urgent need to verify our findings and assumptions about inhalation treatment. Thirdly, extended follow-up could be necessary to demonstrate the long-term impact of AFL on survival after lung surgeries.

\section{Conclusion}

Our study confirmed that asymptomatic patients with AFL are at higher risk of PPCs than the general population in those who had undergone lung surgeries, accompanied by an increase in ICU admissions and 90-day hospital readmissions secondary to PPCs. Although patients with AFL tended to report worse current condition than the normal population, they were similar in the in-hospital, 90-day and overall mortality during the follow-up.

\section{Data Sharing Statement}

We would like to share the individual participant data that underlie the results reported in this article, after deidentification. The study protocol will also be available. The data will be available following article publication. Proposals could be directed to Dr Jinhui Yu yujingui1109@126.com. To gain access, data requestors will need to sign a data access agreement and reasonable requests will be met.

\section{Acknowledgments}

We would like to show our gratitude to all the technicians from the lung function laboratory and Ms. Ma Yanan from the Medical Records Department for their technical support. All the contributors are staff members of Qilu Hospital, Shandong University, China.

\section{Funding}

This work was supported by the National Natural Science Foundation of China [No. 26010105131548].

\section{Disclosure}

The authors declare no conflicts of interest in this work.

\section{References}

1. Vogelmeier C, Criner G, Martinez F, et al. Global strategy for the diagnosis, management, and prevention of chronic obstructive lung disease 2017 report. GOLD executive summary. Am J Respir Crit Care Med. 2017;195(5):557-582. doi:10.1164/rccm.201701-0218PP

2. Datta D, Lahiri B. Preoperative evaluation of patients undergoing lung resection surgery. Chest. 2003;123(6):2096-2103. doi:10.1378/ chest.123.6.2096

3. Wang Y, Xiao W, Ma D, Jiang Y. Predicted lower limit of normal reduces misclassification risk of airflow limitation in asymptomatic elderly never-smokers. Chin Med J. 2013;126(18):3486-3492.

4. Thomsen M, Nordestgaard B, Vestbo J, Lange P. Characteristics and outcomes of chronic obstructive pulmonary disease in never smokers in Denmark: a prospective population study. Lancet Respir Med. 2013;1(7):543-550. doi:10.1016/s2213-2600(13)70137-1

5. Shin S, Park HY, Kim H, et al. Joint effect of airflow limitation and emphysema on postoperative outcomes in early-stage nonsmall cell lung cancer. Eur Respir J. 2016;48(6):1743-1750. doi:10.1183/ 13993003.01148-2016 
6. Xie M, Cui L, Liu J, Wang W, Li J, Xiao W. Impacts of different spirometry reference equations and diagnostic criteria on the frequency of airway obstruction in adult people of North China. Int J Chron Obstruct Pulmon Dis. 2020;15:651-659. doi:10.2147/ copd.S232863

7. Coultas D, Mapel D, Gagnon R, Lydick E. The health impact of undiagnosed airflow obstruction in a national sample of United States adults. Am J Respir Crit Care Med. 2001;164(3):372-377. doi:10.1164/ajrccm.164.3.2004029

8. Singh D, Agusti A, Anzueto A, et al. Global strategy for the diagnosis, management, and prevention of chronic obstructive lung disease: the GOLD science committee report 2019. Eur Respir J. 2019;53(5):1900164. doi:10.1183/13993003.00164-2019

9. Fukuchi Y, Nishimura M, Ichinose M, et al. COPD in Japan: the Nippon COPD epidemiology study. Respirology. 2004;9(4):458-465. doi:10.1111/j.1440-1843.2004.00637.x

10. Agostini P, Cieslik H, Rathinam S, et al. Postoperative pulmonary complications following thoracic surgery: are there any modifiable risk factors? Thorax. 2010;65(9):815-818. doi:10.1136/ thx.2009.123083

11. Jeon JH, Kang $\mathrm{CH}$, Kim H, et al. Video-assisted thoracoscopic lobectomy in non-small-cell lung cancer patients with chronic obstructive pulmonary disease is associated with lower pulmonary complications than open lobectomy: a propensity score-matched analysis. Eur J Cardiothorac Surg. 2013;45(4):640-645. doi:10.1093/ejcts/ezt460

12. Lugg S, Agostini P, Tikka T, et al. Long-term impact of developing a postoperative pulmonary complication after lung surgery. Thorax. 2016;71(2):171-176. doi:10.1136/thoraxjnl-2015-207697

13. Russotto V, Sabate S, Canet J. Development of a prediction model for postoperative pneumonia: a multicentre prospective observational study. Eur J Anaesthesiol. 2019;36(2):93-104. doi:10.1097/ EJA.0000000000000921

14. Abbott TEF, Fowler AJ, Pelosi P, et al. A systematic review and consensus definitions for standardised end-points in perioperative medicine: pulmonary complications. $B r \quad J$ Anaesth. 2018;120 (5):1066-1079. doi:10.1016/j.bja.2018.02.007

15. Canet J, Gallart L, Gomar C, et al. Prediction of postoperative pulmonary complications in a population-based surgical cohort. Anesthesiology. 2010;113(6):1338-1350. doi:10.1097/ALN.0b013e $3181 \mathrm{fc} 6 \mathrm{e} 0 \mathrm{a}$

16. Odor P, Bampoe S, Gilhooly D, Creagh-Brown B, Moonesinghe S. Perioperative interventions for prevention of postoperative pulmonary complications: systematic review and meta-analysis. BMJ. 2020;368:m540. doi:10.1136/bmj.m540

17. Licker MJ, Widikker I, Robert J, et al. Operative mortality and respiratory complications after lung resection for cancer: impact of chronic obstructive pulmonary disease and time trends. Ann Thorac Surg. 2006;81(5):1830-1837. doi:10.1016/j.athoracsur.2005.11.048
18. Sekine Y, Suzuki H, Yamada Y, Koh E, Yoshino I. Severity of chronic obstructive pulmonary disease and its relationship to lung cancer prognosis after surgical resection. Thorac Cardiovasc Surg. 2013;61(2):124-130. doi:10.1055/s-0032-1304543

19. Karrasch S, Brüske I, Smith M, et al. What is the impact of different spirometric criteria on the prevalence of spirometrically defined COPD and its comorbidities? Results from the population-based KORA study. Int $J$ Chron Obstruct Pulmon Dis. 2016;11:1881-1894. doi:10.2147/copd.S104529

20. Pérez-Padilla R, Hallal P, Vázquez-García J, et al. Impact of bronchodilator use on the prevalence of COPD in population-based samples. COPD. 2007;4(2):113-120. doi:10.1080/15412550701341012

21. Arne M, Lisspers K, Ställberg B, et al. How often is diagnosis of COPD confirmed with spirometry? Respir Med. 2010;104 (4):550-556. doi:10.1016/j.rmed.2009.10.023

22. Kronborg T, Hangaard S, Cichosz S, Hejlesen O. Increased accuracy after adjustment of spirometry threshold for diagnosing COPD based on pre-bronchodilator FEV/FVC. Respir Care. 2019;64(1):85-90. doi:10.4187/respcare.06148

23. Fernandez-Bustamante A, Frendl G, Sprung J, et al. Postoperative pulmonary complications, early mortality, and hospital stay following noncardiothoracic surgery: a multicenter study by the perioperative research network investigators. JAMA Surg. 2017;152(2):157-166. doi:10.1001/jamasurg.2016.4065

24. Watanabe S, Asamura H, Suzuki K, Tsuchiya R. Recent results of postoperative mortality for surgical resections in lung cancer. Ann Thorac Surg. 2004;78(3):999-1002; discussion 1002-3. doi:10.1016/ j.athoracsur.2004.04.007

25. Ludwig C, Stoelben E, Olschewski M, Hasse J. Comparison of morbidity, 30-day mortality, and long-term survival after pneumonectomy and sleeve lobectomy for non-small cell lung carcinoma. Ann Thorac Surg. 2005;79(3):968-973. doi:10.1016/j.athoracsur.20 04.08 .062

26. Dyszkiewicz W, Pawlak K, Gasiorowski L. Early post-pneumonectomy complications in the elderly. Eur $J$ Cardiothorac Surg. 2000;17(3):246-250. doi:10.1016/s10107940(00)00339-0

27. Kashiwabara K, Sasaki J, Mori T, Nomori H, Fujii K, Kohrogi H. Relationship between functional preservation after segmentectomy and volume-reduction effects after lobectomy in stage I non-small cell lung cancer patients with emphysema. J Thorac Oncol. 2009;4 (9):1111-1116. doi:10.1097/JTO.0b013e3181ae59e2

International Journal of Chronic Obstructive Pulmonary Disease

Dovepress

\section{Publish your work in this journal}

The International Journal of COPD is an international, peer-reviewed journal of therapeutics and pharmacology focusing on concise rapid reporting of clinical studies and reviews in COPD. Special focus is given to the pathophysiological processes underlying the disease, intervention programs, patient focused education, and self management protocols. This journal is indexed on PubMed Central, MedLine and CAS. The manuscript management system is completely online and includes a very quick and fair peer-review system, which is all easy to use. Visit http://www.dovepress.com/testimonials.php to read real quotes from published authors 